\title{
Interpretation of Polarization Features in Ground-Based Microwave Observations as Caused by Horizontally Aligned Oblate Raindrops
}

\author{
HARALD CZEKALA \\ Department of Applied Physics and Applied Mathematics, Columbia University, and NASA Goddard Institute for Space Studies, \\ New York, New York \\ Susanne Crewell, Clemens Simmer, and Ariane Thiele \\ Meteorologisches Institut der Universität Bonn, Bonn, Germany \\ ACHIM Hornbostel AND ARno Schroth \\ Deutsches Zentrum für Luft- und Raumfahrt, Institut für Kommunikation und Navigation, Oberpfaffenhofen, Germany
}

(Manuscript received 1 September 2000, in final form 17 March 2001)

\begin{abstract}
Based on a comparison of ground-based radiometer measurements with microwave radiative transfer calculations, it is shown that raindrops with an oblate shape and a preferred horizontal orientation have a significant effect on microwave polarization signals when compared with spherical particle shape. Measurements with a dual-polarized $19-\mathrm{GHz}$ radiometer reveal a polarization difference of as much as $-18 \mathrm{~K}$ in the downwelling microwave radiation at $30^{\circ}$ elevation angle. Averaging all rain observations within 19 months leads to a signal of $-6 \mathrm{~K}$. Model calculations covering roughly the same range of weather conditions as that inferred from the meteorological data recorded with the radiometer measurements were carried out with spherical raindrop shape and an oblate particle shape with a fixed horizontal alignment. From the model results, positive polarization difference is expected for spherical particles. This signal was never observed in the recorded data. For oblate drops, the averaged model results lead to a polarization difference of $-8 \mathrm{~K}$, which is in reasonable agreement with the long-term averaged observations. Case studies that compare isolated rain events usually lead to a better match of model and observations. However, there are some major discrepancies in some cases. Possible reasons for the remaining differences are the short-term variations in the cloud microphysics for which the model does not correctly account, such as variations in the melting layer, drop oscillations, or variations in the drop size distribution or angular distribution of the drop alignment. Three-dimensional effects are also important when observing small-scale heavy precipitation. Despite remaining small uncertainties, the comparison presents strong evidence that the oblate raindrop shape, with fixed horizontal alignment, is by far the better choice for accurate radiative transfer calculations than is the spherical shape. The omission of this shape effect can cause significant errors when developing remote sensing algorithms based on model results.
\end{abstract}

\section{Introduction}

Microwave radiative transfer models are a basic foundation of remote sensing applications of the earth and its atmosphere. Sensitivity studies with such models add to general understanding of radiative processes in the earth-atmosphere system. From the results of forward calculations, one may derive retrieval algorithms for a large variety of atmospheric parameters, such as surface temperature, water vapor, cloud liquid water path, and rainfall rate. The accuracy of the forward radiative transfer models is crucial for the quality of derived remote sensing algorithms. For this reason, the existing models,

Corresponding author address: Harald Czekala, NASA Goddard Institute for Space Studies, 2880 Broadway, New York, NY 10025. E-mail: hczekala@giss.nasa.gov which necessarily are still approximations to the complex nature of the atmospheric environment, are subject to revisions and improvement (Liebe 1985; Liebe et al. 1993; Wu and Weinman 1984; Simmer 1994; Turk and Vivekanandan 1995; Evans and Stephens 1995; Haferman et al. 1996; Haferman 1999; Roberti and Kummerow 1999).

Precipitation is an important component of the hydrological cycle, and strong efforts have been put into the retrieval of rain from microwaves. For more than $20 \mathrm{yr}$, the flattened shape of rain particles has been exploited to derive rain rates from polarization-dependent backscattered radar signals (Rinehart 1991; Seliga and Bringi 1976). However, the representation of hydrometeors in current radiative transfer models is mainly justified by mathematical, conceptual, and computa- 
tional limitations rather than by realistic representation of hydrometeors. One of the main reasons for the simplifications is the multiple scattering of polarized microwave radiation by precipitation particles. The size of the hydrometeors is comparable to wavelength, and a straightforward scattering solution for electromagnetic radiation only exists for spherical particles: the LorenzMie scattering theory (Mie 1908).

During the past years, considerable effort has been made to account for more realistic hydrometeor shapes. Wu and Weinman (1984), for example, took into account approximated extinction coefficients for nonspherical ice particles to calculate upwelling microwave radiation. Haferman et al. (1996) considered nonspherical spheroid particles but with random orientation. This random orientation again is not always justified by natural conditions, because falling nonspherical particles will most probably align their largest extension to the horizontal. Random orientation simplifies the scattering calculations by reducing the angular dependence to only the scattering angle between incident and scattered radiation.

Nonspherical particles with a preferred orientation, such as flattened raindrops falling at terminal velocity, complicate the scattering in radiative transfer models. The scattering (as well as extinction, absorption, and emission) caused by such particles depends on explicit propagation directions and needs to be formulated with the four-component Stokes vector formalism to account for the polarized nature of the radiation (Hansen and Travis 1974). Such vector radiative transfer models for oriented nonspherical particles exist only in a small number (Liu et al. 1996; Evans et al. 1998; Haferman et al. 1996; Hornbostel et al. 1995, 1999; Roberti and Kummerow 1999). For a complete review of state-ofthe-art microwave radiative transfer with scattering by nonspherical particles refer to Haferman (1999).

The aim of this study is to show that the inclusion of oblate drop shape with size-dependent deformation and horizontal drop alignment in radiative transfer modeling efforts is well justified by observed data. In addition, we will demonstrate that spherical raindrop shapes totally fail to explain the observations. This evidence of the theoretically predicted polarization signal in radiometric measurements is of importance for future remote sensing techniques that are based on this rainspecific signal (Czekala et al. 2001). Furthermore, this study illustrates that the effect of oblate particle shape introduces (despite varying atmospheric profiles and microphysical details) a significant bias into the polarization signal when compared with calculations assuming spheres, thus emphasizing the importance of using raindrop shapes that are as realistic as possible for all kinds of microwave radiative transfer simulations.

The next section introduces the origin and characteristics of the polarization induced by rain. Section 3 presents the measurement system and the observational dataset. The model results are given in section 4. Section

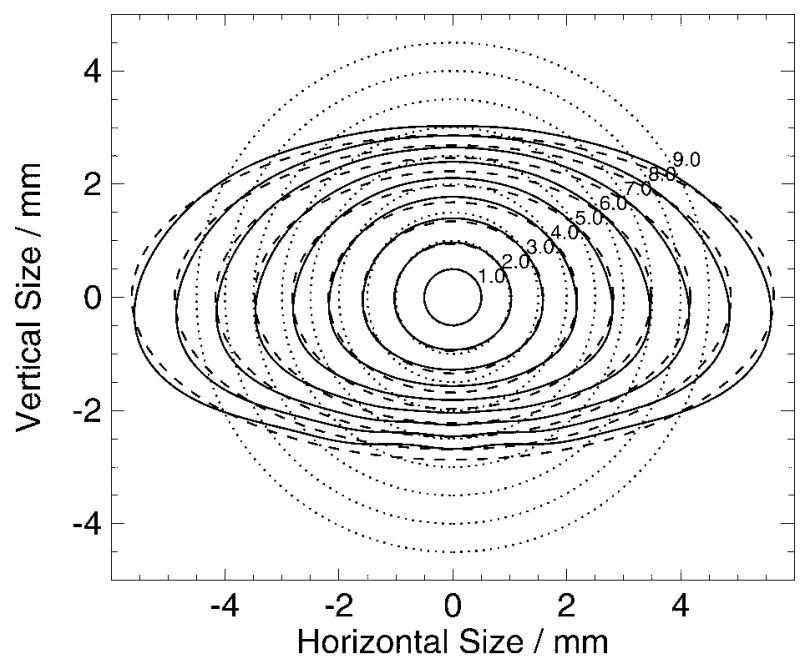

FIG. 1. Shape of raindrops falling at terminal velocity [according to model calculations performed by Chuang and Beard (1990)]. Solid lines give the modeled Chebyshev shapes, dashed lines give the spheroid approximation, and dotted lines indicate the equivalent spherical drop. The numbers within the plot correspond to the drop diameter.

5 gives the comparison and discussion of the predicted and observed results, followed by our conclusions in section 6.

\section{Polarization signal of nonspherical precipitation}

Falling raindrops are known to be nonspherical (Pruppacher and Beard 1970; Pruppacher and Pitter 1971; Beard and Chuang 1987). The shape of drops falling at terminal velocity is modeled by Chuang and Beard (1990) as rotationally symmetric oblate particles with cross sections described by a series of Chebyshev polynomials. The cross section of these shapes is given by the solid lines in Fig. 1.

Our previous investigations (Czekala and Simmer 1998) with a one-dimensional microwave vector radiative transfer model approximated the Chebyshev shapes by spheroids (dashed lines in Fig. 1) of the same volume and same aspect ratio, which is defined as the ratio of horizontal to vertical extension of the drop. Because the shape of falling drops varies with drop size, the aspect ratio of the spheroid drop shape is a function of the drop radius. The drop radius for nonspherical drops is given by the radius of the undisturbed (spherical) drop of the same volume (dotted lines in Fig. 1).

The results of Czekala and Simmer (1998) revealed remarkable differences between spherical and oblate spheroid shapes in the radiative transfer results. The total brightness temperature [TB, defined as the average brightness temperature calculated from the vertically and horizontally polarized brightness temperatures according to $\mathrm{TB}=\left(\mathrm{TB}_{v}+\mathrm{TB}_{h}\right) / 2$ ] showed only a weak dependence on the hydrometeor shape. However, the polarization difference $[\mathrm{PD}$, defined as the difference $\left(\mathrm{TB}_{v}-\mathrm{TB}_{h}\right)$ of the vertically and horizontally polarized 
TABLE 1. Main characteristics of the radiometer.

\begin{tabular}{ll}
\hline \hline \multicolumn{1}{c}{ Feature } & \multicolumn{1}{c}{ Specification } \\
\hline Center frequency & $19.2 \mathrm{GHz}$ \\
Bandwidth & $100 \mathrm{MHz}$ \\
Integration time & $1 \mathrm{~s}$ \\
Sensitivity & $<0.5 \mathrm{~K}$ \\
Noise figure & $5 \mathrm{~dB}$ \\
Antenna beamwidth & $1.2^{\circ}$ \\
Antenna gain & $45 \mathrm{~dB}$ \\
Polarization & $\mathrm{H}, \mathrm{V}(\mathrm{linear}$ orthogonal) \\
Sidelobes & $<-32 \mathrm{~dB}$ \\
Cross polarization & $<-32 \mathrm{~dB}$ \\
Polarization switching & $933 \mathrm{~Hz}$ \\
Dicke switching & $1866 \mathrm{~Hz}$ \\
Dicke reference load temperature & $323 \pm 0.2 \mathrm{~K}$ \\
Elevation & Fixed $\left(30^{\circ}\right)$ \\
Azimuth & Fixed $\left(180^{\circ}\right)$ \\
\hline
\end{tabular}

brightness temperatures] for downwelling radiation was altered from small positive values in the case of spherical raindrops to large negative values in case of oblate spheroids. The precise amount of negative PD varied with the optical thickness within the observed volume: the amount of precipitation, cloud-top and cloud-base heights, the chosen frequency, the atmospheric temperature, and the elevation angle of the hypothetical ground-based observation controlled the amount of PD predicted by the radiative transfer model.

A more recent study (Czekala et al. 1999) used the more realistic Chebyshev shapes for the raindrop shapes instead of the spheroid approximation. For liquid hydrometeors and microwave frequencies below $100 \mathrm{GHz}$, the more realistic shapes produced results very similar to the spheroid approximations. Only for ice particle scattering did the deviation of the approximated shapes from the detailed shapes become more important, but still the main effect of nonsphericity was described well by spheroids of the equivalent aspect ratio.

\section{Observational data}

\section{a. Description of the radiometer}

The measurements were performed in southern Germany with a 19-GHz dual-polarization Dicke radiometer that has been operated by the Deutsches Zentrum für Luft- und Raumfahrt (DLR) since 1990. The main instrument characteristics are presented in Table 1. The receiver front end is mounted directly to the antenna horn. By use of an orthomode transducer, the brightness temperature is measured in two linear orthogonal polarizations. Two electronically controlled ferrite circulators switch permanently between the two polarization channels and between the antenna entrance and the internal Dicke reference load. The effective switching frequencies are 933 and $1866 \mathrm{~Hz}$, respectively. In standard operation mode, the brightness temperature is measured in vertical and horizontal linear polarization. Measurements demonstrated an excellent linearity between the detector output voltage and the antenna input temperature. The calibration was performed in two ways: either by use of external reference loads, which were connected directly to the receiver front end, or by measuring the antenna temperatures during clear-sky conditions and the temperature of a blackbody absorber placed in front of the antenna horn. Because of the less technical effort needed, the second method was applied more frequently. For the polarization difference, this method is even more accurate, where as the first method is preferable with respect to absolute accuracy. Calibration checks by tipping-curve measurements showed that the achievable calibration accuracy is about $1 \mathrm{~K}$ for the measured brightness temperatures. For the polarization difference, an accuracy of $0.2 \mathrm{~K}$ could be reached by further data processing (see section $3 b$ ). The calibration coefficients have a very good long-term stability due to the efficient temperature control inside the front end, which keeps the inner box temperature and the Dicke reference load temperature constant at $323 \pm 0.2 \mathrm{~K}$. Further technical details about the instrument and the calibration procedures are described in Hornbostel and Schroth (1995).

Raindrops on the radome (thin Kapton film) of the antenna horn were a severe problem at the beginning of the measurements. We observed that such drops produced large spikes in the polarization difference that had normally higher values and the opposite sign than the polarization difference caused by rain in the path and therefore could be detected easily. These drops were not removed by a warm air blower, which was first applied alone. Therefore, a small Perspex roof was constructed and installed directly above the antenna horn in such a way that it did not influence the antenna characteristics. The roof kept the horn completely dry, and the spikes never appeared again. All measurements analyzed in this paper were performed after installation of the roof.

Jacobson et al. (1986) found that the existence of a water film on the antenna reflector can also produce polarization effects, which can disturb the measurements. However, there are some differences between the measurement setup in this paper and the DLR radiometer. Jacobson et al. (1986) used a reflector with an alignment of $45^{\circ}$. The DLR radiometer has an asymmetric Cassegrain antenna with a beamwidth of $1.2^{\circ}$ full with half maximum (see Fig. 2). Because of the antenna asymmetry, the alignment of the main reflector and subreflector is nearly vertical, so water can flow up quickly. Both reflectors are heated to avoid icing and to speed up the removal of drops.

Another difference is that Jacobson et al. (1986) investigated a radiometer that uses two different frequencies $(20.6$ and $31.65 \mathrm{GHz}$ ) for separation of the two polarization channels. Their modeled and measured curves show that the polarization difference due to reflector wetness at these two frequencies is significantly higher than the difference at the single $20-\mathrm{GHz}$ fre- 


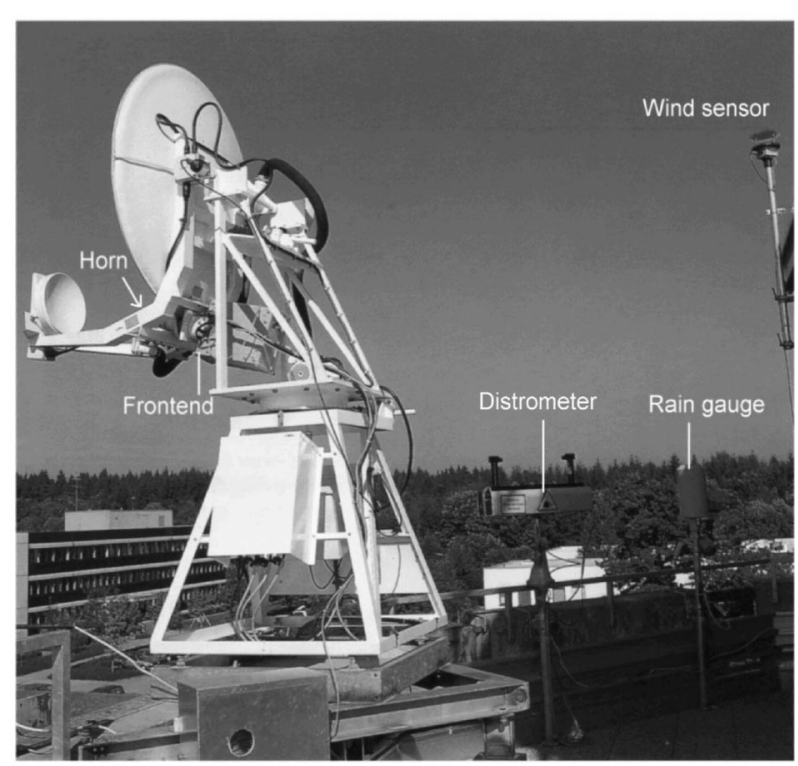

FIG. 2. Photograph of the DLR radiometer and associated instruments, described in section 3 .

quency, at least for water films of up to $0.2-\mathrm{mm}$ thickness. Although the presence of drops on the reflectors could certainly not be avoided, we never observed the development of a continuous water film.

There are some further experimental experiences, from which we conclude that wetness of the reflector did not significantly disturb our measurements:

1) At the antenna site, a $13-\mathrm{GHz}$ radiometer was installed that looked into the same direction as the 19$\mathrm{GHz}$ radiometer but was a completely independent system. It had a simple horn antenna, which was also protected by a Perspex roof. An example of concurrent measurements by both instruments is presented in Fig. 3. Although the $13-\mathrm{GHz}$ radiometer had a different beamwidth $\left(12^{\circ}\right.$ in contrast to $1.2^{\circ}$ for $19 \mathrm{GHz}$ ) and was difficult to calibrate with respect to long-term and temperature stability, the measured polarization difference at $13 \mathrm{GHz}$ shows qualitatively the same behavior versus time as does the 19-GHz polarization difference. Another example of these concurrent multifrequency measurements is shown by Kutuza et al. (1998).

2) Another interesting fact in Fig. 3 is that the increase of rain rate at the antenna location starts earlier than the increase of brightness temperature and polarization difference; that is, the rain cell was moving from the direction of the antenna site (from north) into the path (to south). At the beginning of the rain event, when the antenna must have been wet already but the main part of the radiometer path was still free of rain, practically no change in the polarization difference is observed. There were also opposite situations in other rain events in which the rainfall occurred first somewhere in the radiometer path- for example, in a distance 1 or $2 \mathrm{~km}$ apart from the antenna-when directly at the antenna site no rain was recorded or it was recorded later. An increase of brightness temperature and negative polarization differences was also observed in these conditions, that is, with a dry antenna. In summary, we often observed a time lag between the rain rate at the antenna site and the increase of brightness temperature. However, the measured polarization differences were always temporally correlated with the brightness temperature but not with the rain rate. In contrast, disturbances due to antenna wetness should be temporally correlated with the rain rate at the antenna site.

3) During some rain events, the radiometer path was scanned additionally by a dual-polarization C-band weather radar. The radar reflectivity and differential reflectivity measurements confirmed the rain polarization signatures obtained by the radiometer measurements (Hornbostel et al. 1997).

4) In winter, when snow flakes melted on the heated antenna reflectors, the polarization difference still was small, even in cases in which the brightness temperature had significant values during intense fall of wet snow.

\section{b. Data recording and processing}

To find physical evidence for the negative polarization differences produced by the radiative transfer model using oblate horizontally aligned water drops, we analyze observations from the $19-\mathrm{GHz}$ DLR radiometer and corresponding rain gauge data. Observations were made during 5 months in 1996 and continuously from November of 1998 through December of 1999. System maintenance and sensor failures caused some missing days in the time series (Table 2). Observations from 513 days within 19 different months were available for this study.

The south-looking viewing geometry of the radiometer matches with the main southern wind direction at the observing site but causes some problems in certain (very rare) cases: during some days in spring and autumn (around 19 February and 22 October), the small antenna beam is pointing toward the noontime sun for several minutes. These observations of up to $600 \mathrm{~K}$ had to be rejected by removing $1 \mathrm{hr}$ of data for the weeks before and after these dates.

Downwelling brightness temperatures at vertical and horizontal polarizations were recorded simultaneously. Together with the radiometer observations, the ambient meteorological parameters [rain rate RR, pressure, relative humidity, wind speed, and wind direction] were recorded at the position of the radiometer at every 10 $\mathrm{s}$. The radiometer measurements were also recorded every $10 \mathrm{~s}$ with an absolute accuracy of less than $1 \mathrm{~K}$, but the polarization difference can be calibrated by clear- 

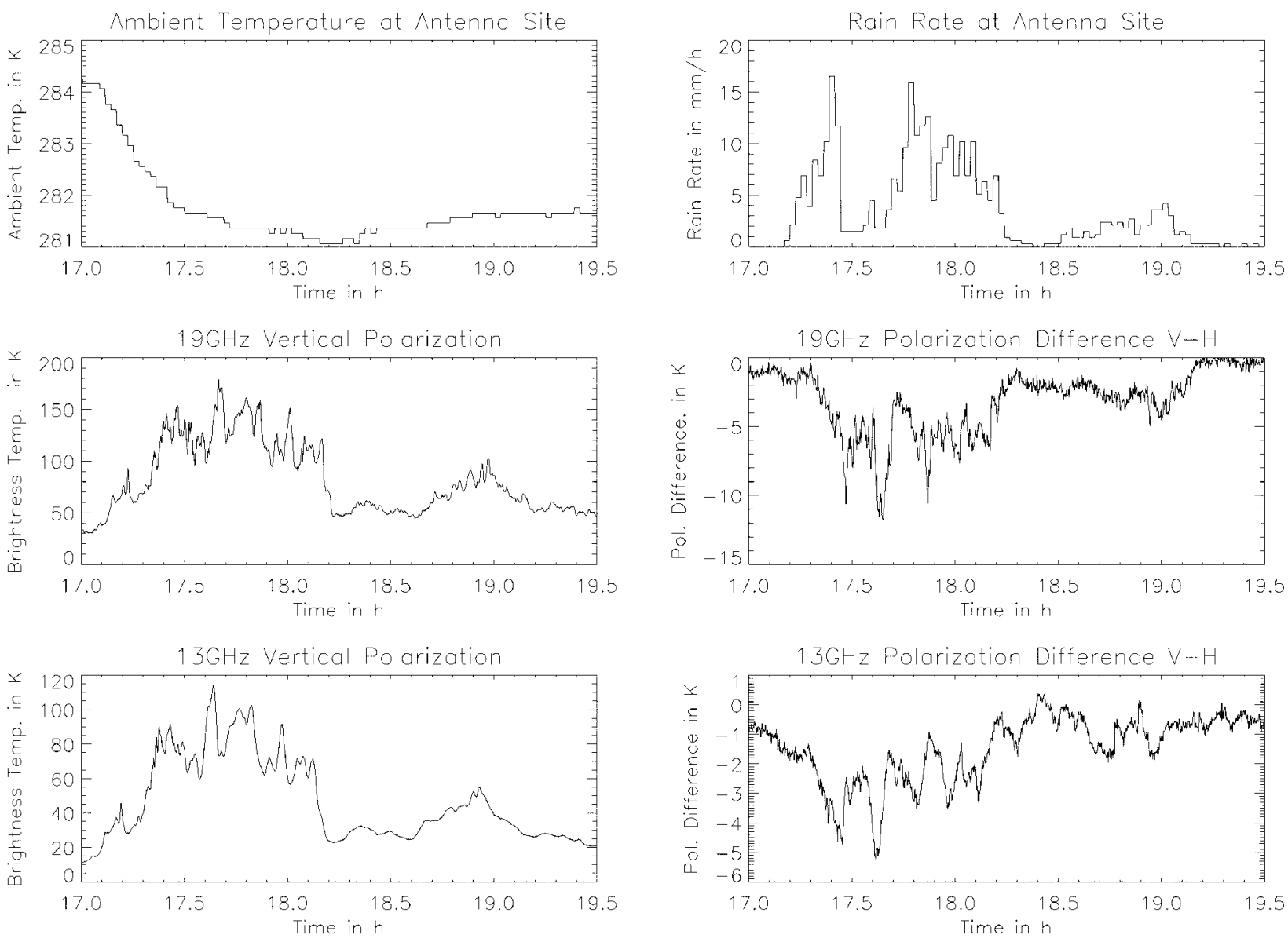

FIG. 3. Comparison of temperature, rain rate, brightness temperature, and polarization difference at 19 and $13 \mathrm{GHz}$ (14 Sep 1996).

sky observations so that the relative accuracy of the PD measurement is approximately $0.2 \mathrm{~K}$.

The low elevation angle of $30^{\circ}$ is suited well for the observation of large negative $\mathrm{PD}$ at $19 \mathrm{GHz}$ but causes a problem of matching the surface rain rate to the radiation data because of the different sampling volumes of the instruments. Only for a directly upward looking

TABLE 2. Temporal coverage of the data available from the 19$\mathrm{GHz}$ radiometer of the DLR Oberpfaffenhofen.

\begin{tabular}{lccc}
\hline \hline & \multicolumn{3}{c}{ Observed days per month } \\
\cline { 2 - 4 } Month & 1996 & 1998 & 1999 \\
\hline Jan & - & - & 27 \\
Feb & - & - & 25 \\
Mar & - & - & 31 \\
Apr & 10 & - & 28 \\
May & - & - & 29 \\
Jun & - & - & 29 \\
Jul & 31 & - & 29 \\
Aug & - & - & 31 \\
Sep & 21 & 14 & 30 \\
Oct & 31 & 31 & 31 \\
Nov & 25 & & 30 \\
Dec & - & & 30 \\
\hline
\end{tabular}

radiometer does the observed RR correspond to the observed radiation measurement. With an inclined optical path, the detected radiation originates from a volume along the line of sight, leading to radiometric observations of precipitation that either are never recorded by the rain gauge or may be observed at different observation times, shifted positively or negatively with respect to the radiometer data. This time shift corresponds to displacements of as much as $2 \mathrm{~km}$ from the radiometer position.

The time lag between radiometric observation of precipitation and the recording at the rain gauge is zero only when the cloud structures are homogeneous in time and space. Otherwise the time lag depends on wind direction, wind speed, and the average height from which the radiation originates. In addition, a beginning rain event will not be recorded immediately but rather with a time lag that depends on rain intensity and the type of detection device. This instrumental delay adds to the geometric delay and also needs to be corrected.

The time lag (determined by a procedure described below) reaches up to $10 \mathrm{~min}$ for typical situations with slowly varying precipitation evolution and wind directions that have a significant component in the north- 
south direction. For wind directions perpendicular to the pointing direction and nonhomogeneous cloud fields, there will be no physical connection between observed rain rates and brightness temperatures. The same is true for inhomogeneous rain events that vary on timescales smaller than the time or the movement of a cloud from the rain gauge position to the field of view of the antenna.

Because we have to make sure that the observed rain rates and brightness temperatures originate from the same physical rain event, the data need to be preprocessed. This filtering has to rely on meteorological data (RR, pressure, temperature, humidity, and wind) because other sources of information (e.g., radar) were not available with an appropriate temporal coverage.

Calculation of the time lag from wind direction and wind speed failed because the average rain-layer height could not be determined with sufficient accuracy from the meteorological data at the surface. Another reason for a failing analytical determination of the time lag may be the imperfect correlation between surface winds and winds at the cloud height. Instead, an empirical approach was chosen: the correlation between brightness temperature and rain rate was maximized to determine the time lag. From the available data (513 days; see Table 2) those 262 days were selected that show a nonzero surface rain rate at least once a day.

To correct for errors in the absolute calibration, a recalibration procedure was applied to the brightness temperatures prior to any filtering. From sequences that are unambiguously identified as rain free (from the observed $R R$ and the TB) the average polarization difference $\mathrm{PD}_{\text {clear }}$ was calculated for each day. Because for clear-sky conditions the downwelling radiation is unpolarized, the $\mathrm{TB}_{v}$ and $\mathrm{TB}_{h}$ values are adjusted by plus and minus $0.5 \mathrm{PD}_{\text {clear }}$, respectively, to obtain zero PD for clear (nonraining) cases. The adjustments were below $1 \mathrm{~K}$ at all the times. The total observation time was $6255 \mathrm{~h}$ from 262 rainy days. The small size of this polarization adjustment gives additional confidence that the polarization signal is not seriously contaminated by effects of a wet antenna, because a short rain event would lead to a change of the polarization signal after the rain shower had passed and the antenna is still wet.

Following the calibration, all observed time series of RR and TB were divided into sequences of 1-h subsets. These subsets were shifted in time from $+25 \mathrm{~min}$ to $-25 \mathrm{~min}$ in time steps of $10 \mathrm{~s}$. At each step, the correlation of RR with TB was calculated. The correlation of RR and TB as a function of time lag was used subsequently for the data processing: if a maximum correlation above a threshold of 0.75 was reached, the center of this peak in the correlation function was determined and was used for shifting the TB observations in time. The overlapping part of the radiometric and rain time series was used as matched data. For each 10$\mathrm{s}$ interval of the matched data, a trio of numbers (RR, $\mathrm{TB}$, and $\mathrm{PD}$ ) was kept for further investigations. If the correlation did not reach a maximum above the desired threshold within the maximum time shift of $\pm 25 \mathrm{~min}$, the 1-h subsets were rejected and were not used for further processing. Overall, $422 \mathrm{~h}$ of matched rain observations could be used for further data processing, which is $6.7 \%$ of of the total time of all rainy days. The magnitude of this number is due to several reasons: 1) rain events of only some minutes as well as 24-h may occur during a day - the segmentation into hours rejects all nonraining hours and therefore reduces the available observation time; 2) the correlation between rain rate and brightness temperature will be destroyed by inhomogeneous cloud structures moving in directions other than north or south (this is very likely to occur during the year); 3) if the precipitation event changes too fast, the correlation between TB and RR will be lost and the observation is not useful for our study; and 4) even with homogeneous conditions in time and space, we would not expect perfect correlation of TB and RR.

Given the possibilities that tend to destroy the correlation between TB and RR in our observations, the remaining amount of data with a correlation of at least 0.75 is large. After the matchup process, the remaining data still cover 166 different days within all 18 months that contained rainy days. The sample of $422 \mathrm{~h}$ (with 10 -s time resolution) gives a representative database of those cases for which the observed microwave radiation actually refers to the observed rain rate.

The reason for using this correlation technique when calculating the time lag between rain and radiometer recordings is that a clear signal of liquid water and precipitation is expected when looking at the observed TB. If there is no increase of TB with increasing rain intensity and if the pattern of TB time series cannot be matched to the RR time series with a good correlation within a small time shift of up to $25 \mathrm{~min}$, then we have to conclude that the radiometer simply has not observed the same rain event that was recorded by the rain gauge, making a comparison of polarization signal and rain rate impossible.

Note that the correlation of TB with RR as a filter criterion does not predetermine the results that we will obtain when comparing measured and modeled PD for those selected cases. We use the well-known dependence of TB on RR to identify those observations for which emerging radiation is actually coupled with the measured rain event. For those points, we compare the observed PD with the theoretical predictions made by the radiative transfer model for both spherical and nonspherical raindrop shapes.

From the matched data, the brightness temperature TB and the polarization difference PD are plotted as functions of RR. For this purpose, we sorted the data into classes of rain rates, each with a width of $1 \mathrm{~mm}$ $\mathrm{h}^{-1}$. From all observations that fall, for example, into a class from 4.5 to $5.5 \mathrm{~mm} \mathrm{~h}^{-1}$, the average TB and PD together with the standard deviations are calculated and then are indicated as the result for $5 \mathrm{~mm} \mathrm{~h}^{-1}$ rain rate. 

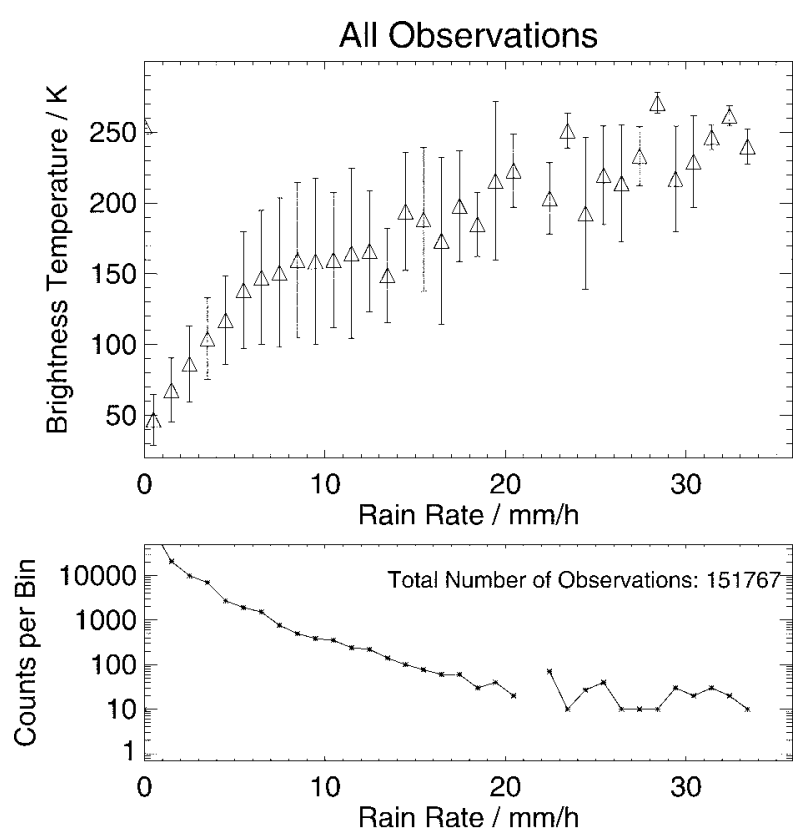

FIG. 4. Observed TB vs rain rate for all observations. The observations are averaged within intervals of $1 \mathrm{~mm} \mathrm{~h}^{-1}$ rain rate. (top) The average TB and its standard deviation; (bottom) the number of observations used for the averaging.

Figures 4 and 5 show the resulting mean values and standard deviations for all classes of rain rates. The number of observations from which mean values and standard deviations were calculated are shown in the lower panels of each figure. Although some key features expected from the model results of Czekala and Simmer (1998) are reproduced by the observations (such as increasing TB with increasing RR, decreasing PD to negative values with small RR), the results show a larger variation at rain rates above $15 \mathrm{~mm} \mathrm{~h}^{-1}$.

It is obvious from Fig. 4 that the brightness temperature is not strictly linked to the surface rain rate, especially at higher rain rates. The PD signal is nearly constant between a 5 and a $15 \mathrm{~mm} \mathrm{~h}^{-1}$ rain rate and is scattered at higher rain rates. However, this behavior does not imply that the relationship between RR and $\mathrm{PD}$ is generally unpredictable at higher rain rates. Instead, this feature arises from the problem of unknown vertical distribution of the rain rate and especially the unknown liquid water path (LWP). The dependence (as derived from radiative transfer calculations) of TB and $\mathrm{PD}$ on the total amount of liquid water along the line of sight is more pronounced than the influence of the drop size distribution or the actual vertical distribution of the hydrometeors.

At higher altitudes, rain particles are formed from either collision-coalescence of cloud droplets or more often at midlatitudes from ice particles (leading also to particles with mixed phase at certain altitudes, specifically in the melting layer). At lower altitudes, evaporation effects can also become significant and reduce
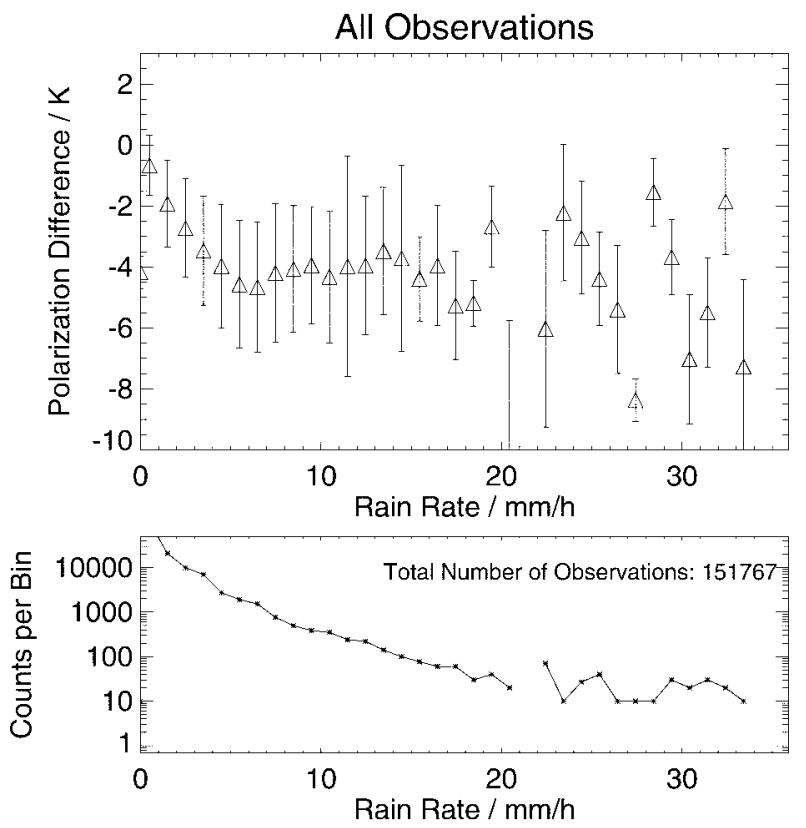

FIG. 5. Observed PD vs rain rate for all observations. The averaging process is similar to that in Fig. 4.

the rain rate again. In addition to the microphysical processes, the atmospheric dynamics (e.g., the vertical wind) can also modify the rain-rate profile, leading to a multitude of possible atmospheric states. If we assume a constant rain rate with height, then a comparison of only those cases with a similar rain-layer height would show a more obvious dependence of TB and $\mathrm{PD}$ on rain rate. Because we use observations from many different days, the rain-layer height varies and leads to this irregular behavior at higher rain rates.

From ground-based observations alone, we cannot reconstruct the rain-top height or even the melting-layer height with sufficient accuracy. However, at $19 \mathrm{GHz}$, the atmosphere is transparent enough to propagate radiation from the complete atmospheric path toward the radiometer. As a consequence, the TB measurements are linked to the total LWP along the radiometer's line of sight and not to the surface rain rate. Because we were using data from 166 days of observations from all seasons of the year with very different types of precipitation and rain-layer heights, we had to work around this source of ambiguity. The most efficient and obvious method of compensation is the elimination of the explicit rain-rate dependence in Figs. 4 and 5 by making PD a function of TB. Both signals are expected to show a strong correlation with the amount of rain water in the field of view. So, we will only look at the amount of polarization that is observed at specific brightness temperature amounts (Fig. 6). In this graph, we present the result for all matched data after the correlation process, thus covering all seasons within the year and all kinds of atmospheric conditions. This time the data trios from Figs. 4 and 5 are sorted and averaged into classes 


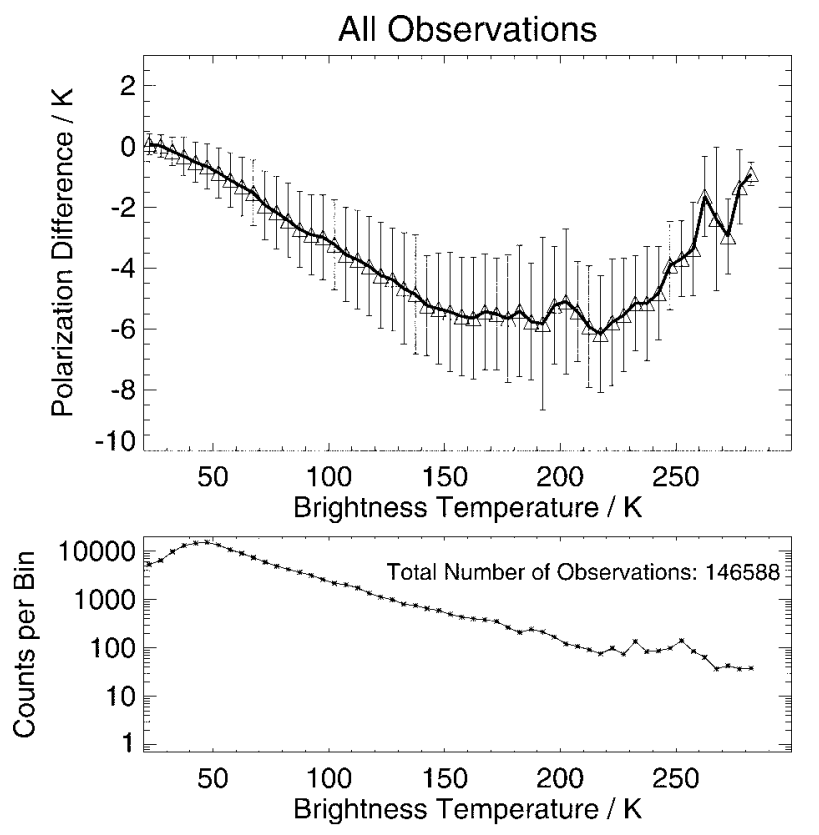

FIG. 6. (top) Observed PD vs TB. The data have been sorted into classes of $5 \mathrm{~K}$ within TB. For each class, the average PD and the standard deviation are given. (bottom) The number of observations within each class.

of TB with a width of $5 \mathrm{~K}$. With increasing TB (caused by increasing LWP), the PD first drops almost linearly to negative values near $-6 \mathrm{~K}$ and then starts to saturate at higher brightness temperatures of roughly 150-220 $\mathrm{K}$. Beyond $220 \mathrm{~K}$, the PD signal decreases in amplitude and approaches zero PD. The fully saturated TB signal for totally opaque atmospheric conditions reaches the ambient temperature $(290 \mathrm{~K})$ at rain rates between 35 and $40 \mathrm{~mm} \mathrm{~h}^{-1}$. The standard deviation for the PD results is smallest for small TB (e.g., $50 \mathrm{~K}$, corresponding to a small LWP) and roughly doubles with increasing LWP.

The results in Fig. 6 are an average over a long observation period. The large amount of data allows for a complete coverage of nearly all possible TB values, up to the maximum $280-\mathrm{K}$ brightness temperature. Shorter observational periods produce results that are less complete but lack the averaging and therefore show the polarization signal of a single rain event. Figure 7 gives the rain event from 8 June 1999. The PD linearly drops to the point of saturation at $150 \mathrm{~K}$. The minimum $\mathrm{PD}$ is now $-8 \mathrm{~K}$ instead of $-6 \mathrm{~K}$ for the averaged results. Because only one specific weather condition is considered, the standard deviation of the results is much smaller than in the long-term average. Comparing the number of averaged observations in Figs. 6 and 7 indicates that the errors shown in Fig. 6 do not represent measurement errors expected when observing a specific rain event. With mean values calculated from less than 100 observations, the standard deviation in Fig. 7 is always smaller when compared with the average over

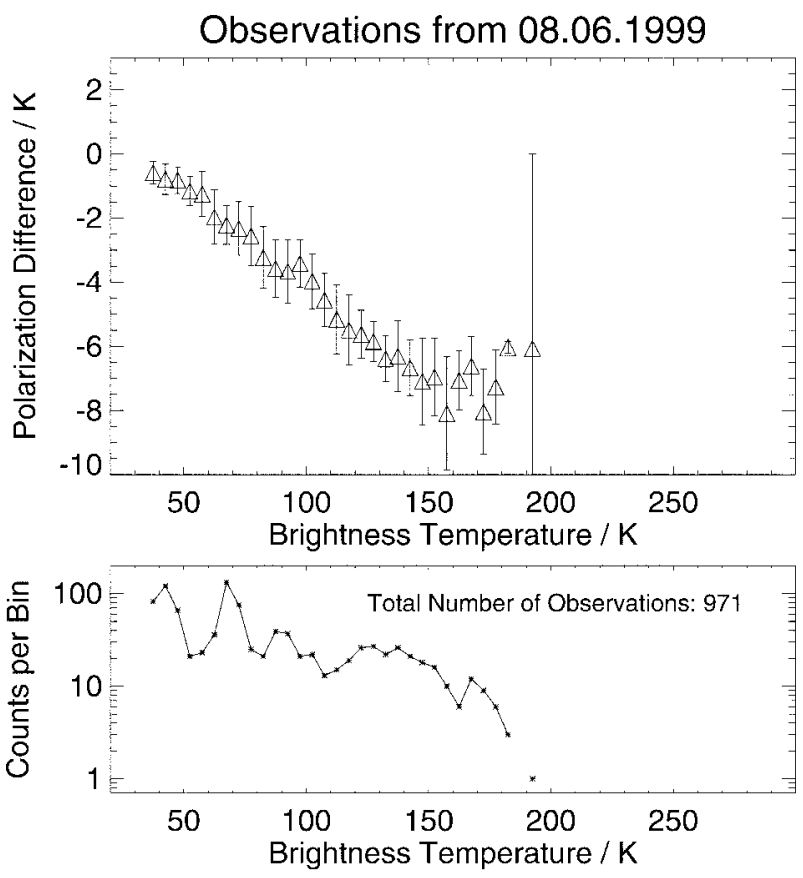

FIG. 7. Observed PD versus TB for 8 Jun 1999 (as in Fig. 6, but with data from only 1 day).

all observations. Thus the increasing standard deviation in Fig. 6 originates from averaging single observations with slightly different slopes and minimum polarization differences at about 200-K brightness temperature.

Figure 8 further illustrates the possible sources of the large standard deviation at the saturation point of the
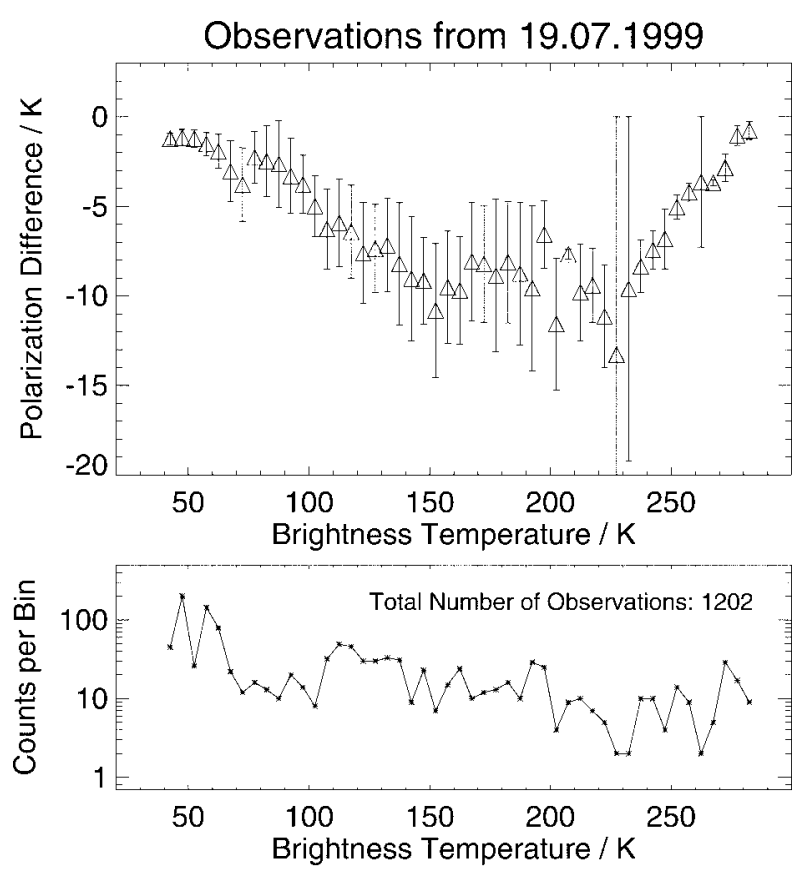

FIG. 8. Observed PD vs TB for 19 Jul 1999 (as in Fig. 6, but with data from only 1 day). 


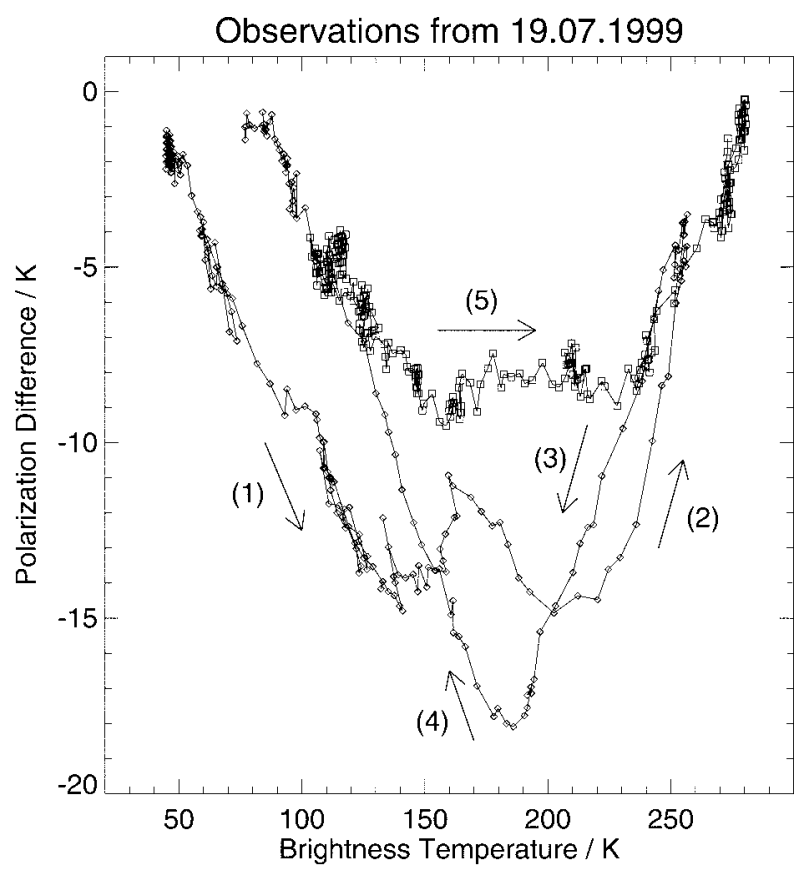

FIG. 9. Observed PD vs TB for two different rain events on $19 \mathrm{Jul}$ 1999 (using the raw data from Fig. 8, but without sorting the data into classes and omitting data with small rain rates between the two rain events). Arrows 1-4 indicate the evolution in time of the first event and arrow 5 indicates the second event, illustrating the variability caused by variations of viewing geometry and size distribution.

PD signal. The matched data from 19 July 1999 are presented. Rain rates of up to $33 \mathrm{~mm} \mathrm{~h}^{-1}$ were recorded, leading to a coverage of the whole TB range from 50 to $280 \mathrm{~K}$. The minimum PD drops as low as $-10 \mathrm{~K}$ for the average values but shows larger standard deviations than the average over 166 days (Fig. 6). This result is caused by observing at least two very different rain events on the same day. When plotting the single observations from this day without averaging (Fig. 9) the two rain events can be identified. The subsequent observations are connected with lines, showing the evolution of the rainfall with time. The spacing between the points corresponds to $10 \mathrm{~s}$. The first rain event (diamond symbols, arrows 1 to 4 give the sequence in time) reaches negative $\mathrm{PD}$ of down to $-18 \mathrm{~K}$ and reveals a slightly different behavior for the first half (increasing rain rate from zero to maximum rain rate, arrows 1 and 2) as compared with the second half (decreasing rain rates from maximum back to zero, arrows 3 and 4). The second rain event (square symbols, arrow 5) reaches only down to $-9 \mathrm{~K}$ but shows a slightly larger saturated TB (280 instead of $255 \mathrm{~K}$ ). These two events differ mainly in temperature (and thus in the rain-layer/melting-layer height) and the relative humidity. Whereas the second event (arrow 5) is associated with temperatures of $15.2^{\circ}-16.5^{\circ} \mathrm{C}$ and a relative humidity that is always larger than $95 \%$, the first event with the more dominant polarization signal is associated with temperatures of $23^{\circ}-29^{\circ} \mathrm{C}$. The rain rates are also higher but are not continuously distributed up to the maximum of $36 \mathrm{~mm}$ $\mathrm{h}^{-1}$. The relative humidity is mostly between $50 \%$ and 95\%. In summary, the large PD response may be due to a very short-lived and small-scale thunderstorm precipitation event that moved into the radiometer's field of view. In such a case, only a small section of the entire radiometer beam would be pointing into raining areas.

\section{Model calculations}

The aim of the following model calculations is to account for the variability of atmospheric conditions that occurred during the observation period. The resulting model output of PD and TB at a given RR is then processed similarly to the experimental data. The one-dimensional vector radiative transfer model used in this study is described in Czekala and Simmer (1998) and Czekala et al. (1999). The shape of oblate raindrops is approximated by rotationally symmetric oblate spheroids (Fig. 1). The particles are perfectly aligned with their rotational axis to the vertical. For single-scattering calculations, the T-Matrix code by Mishchenko was used (Mishchenko et al. 1999; Mishchenko 2000).

To cover a similar variety of atmospheric conditions as were found in the observations, we generated 720 atmospheric profiles by varying the atmospheric temperature profile (six cases), the rain-layer height (six cases), and the rain rate (20 cases). Temperature profiles for model calculations are derived from the near-surface temperature by assuming a vertical profile of relative humidity (consistent with the cloud-top height) and the corresponding atmospheric lapse rates. Six different near-surface temperatures $\left(0^{\circ}, 5^{\circ}, 10^{\circ}, 15^{\circ}, 20^{\circ}\right.$, and $25^{\circ} \mathrm{C}$ ) were chosen during the model calculations, resulting in six different gas absorption profiles that cover the variation of atmospheric conditions during the observing period.

The variation of precipitation amount within the field of view was modeled by introducing raining layers into the model atmospheres with varying vertical extension. The rain layers start from the surface but may reach different top altitudes from 0.5 to $3 \mathrm{~km}$ in steps of 500 $\mathrm{m}$. A vertically constant rain rate was used. The calculations were repeated for varying rain rates between 0 and $50 \mathrm{~mm} \mathrm{~h}^{-1}$ and for both spherical and oblate raindrop shapes. A Marshall-Palmer drop size distribution with rain-rate-dependent parameters is assumed. Nonspherical drops were identified by the radius of the spherical drop with equivalent volume. The surface emissivity was set to 0.9 for all cases. The angular resolution was defined by a set of Gaussian angles, with 10 angles per hemisphere.

For comparing the previously presented observations with model data, we chose a subset from this set of calculations that matches the meteorological conditions that occur in the observation period. Histograms of the data used in Fig. 6 show that the vast majority of data is associated with temperatures between 273 and $293 \mathrm{~K}$ 


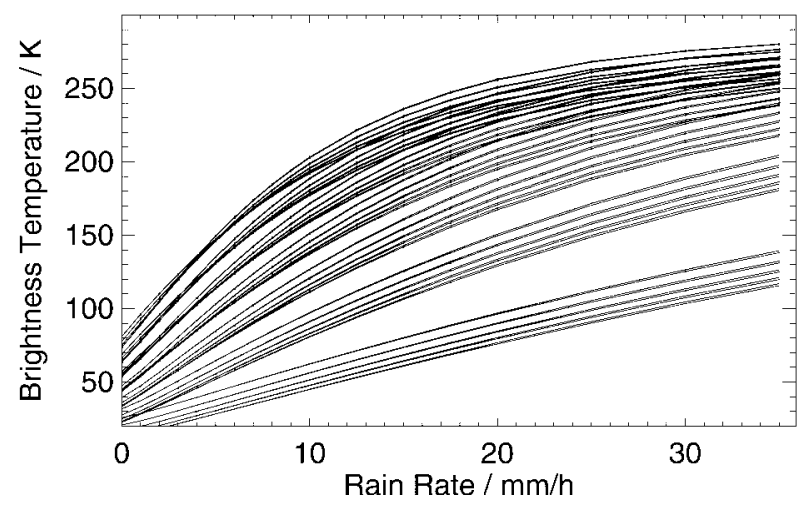

FIG. 10. Range of model-calculated TB as a function of rain rate. Rain rate increases along the lines. Atmospheric temperature has a small impact on $\mathrm{TB}$; the increasing rain-layer heights introduce a much more pronounced increase in TB. There is no observable difference between calculations using spherical and oblate particles.

and with relative humidity larger than $90 \%$. The rain rate reaches up to $35 \mathrm{~mm} \mathrm{~h}^{-1}$, not only with a large peak rain rate but also covering nearly continuously all intermediate rain rates. The major problem when selecting the appropriate model output for comparison is estimating the rain-layer height. As an upper limit we infer the melting-layer height from the measured surface temperature by applying a constant lapse rate of $7 \mathrm{~K}$ $\mathrm{km}^{-1}$.

The constrained model results are shown in Figs. 10 and 11. Figure 10 illustrates the covered range of the brightness temperature as a function of rain rate. Figure 11 illustrates the PD as a function of rain rate. Both graphs reveal a strong impact of rain-layer height on the TB and PD results: TB is increased with increasing vertical extension of the raining area. This effect is stronger than the influence of atmospheric temperature on TB. The temperature effect of TB is more pronounced for optically thin atmospheres with small rainlayer height and small TB. With respect to TB, there are almost no differences between spherical results and nonspherical results. The TB increases with rain rate and rain-layer thickness; saturation of TB with largest LWP amounts is caused by rain rate or rain-layer thickness.

However, the PD-versus-RR graph is clearly divided into two branches. Spherical particles result in small positive PD and vanishing variation with RR and rainlayer thickness. Oblate drops within thin rain layers produce an almost linear decrease of PD with RR until saturation. The saturation is due to increased optical thickness, which is damping the PD generation. The sensitivity of PD to RR (e.g., the slope of the PD-versus$R R$ curve) is increased for thicker rain layers because of the increased amount of water mass (resulting in higher absolute numbers of nonspherical particles). This increased sensitivity saturates at increasing rain rates, with saturation reached at lower rain rates for larger rain-layer thickness.

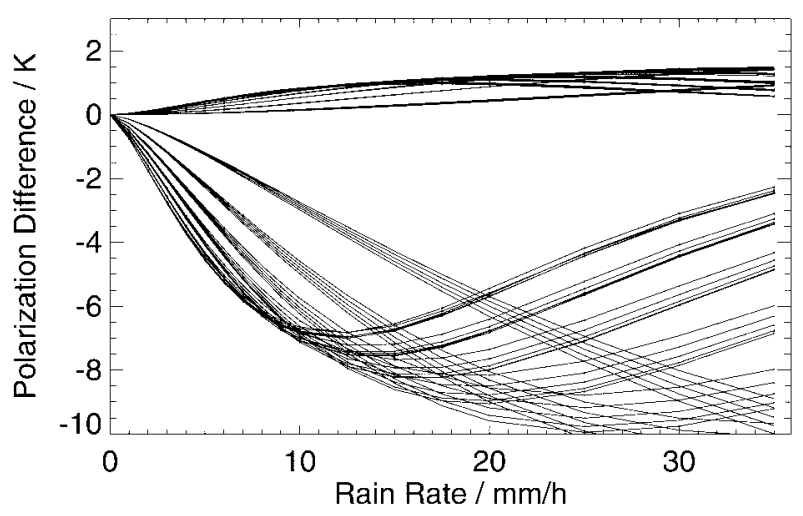

FIG. 11. Range of model-calculated PD as a function of rain rate. All results with positive PD are for spherical particles; all negative PD results are for oblate particles. Smaller initial slopes (at small rain rates) correspond to smaller rain-layer heights. Atmospheric temperature has a smaller impact on the results than does the rain-layer height.

Combining Figs. 10 and 11 into Fig. 12 with PD as a function of $\mathrm{TB}$ produces a more unified behavior of the polarization signal. Each line in this graph represents a fixed temperature profile and a fixed rain-layer height. Along the lines, the rain rates increase from left to right. One important feature of Fig. 12 is the fact that prior to saturation at higher rain rates all lines are mostly parallel. This means that the sensitivity of PD with increasing TB is very similar for all conditions as long as the signal remains unsaturated. However, the exact resulting TB is determined by the total LWP within the field of view. This fact causes a shift along the TB axis with increasing rain-layer-top height: largest top heights reveal largest TB values. So, the major shift in TB at moderate rain rates is associated with the rain-layer height. In contrast to this observation, TB for saturated signals in optically thick situations (right end of Fig. 12) is primarily determined by the atmospheric temperature.

For further comparison, we applied the same histogram process with $\mathrm{TB}$ classes of $5-\mathrm{K}$ width (also used

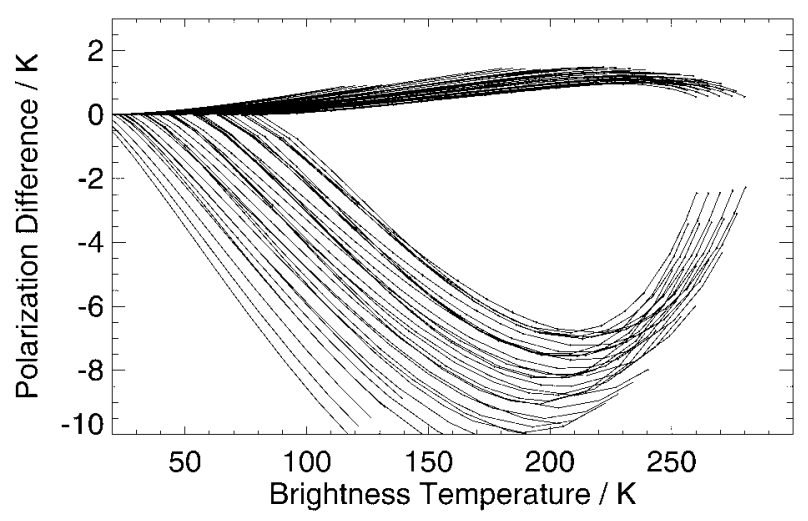

FIG. 12. Combination of model-calculated PD and TB (from Figs. 10 and 11). For details see text. 


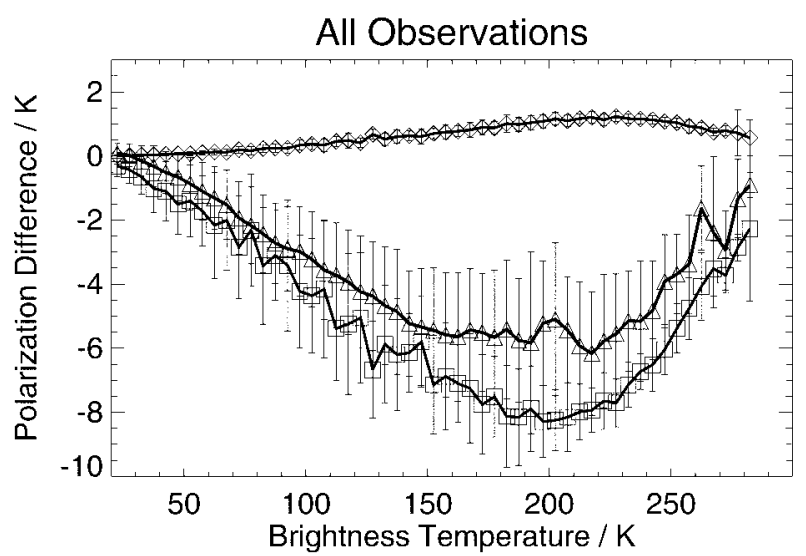

FIG. 13. Model-calculated PD as a function of TB in histogram mode (same data as in Fig. 12, but with mean values within 5-K classes). Diamonds (upper line) are used for spherical particle shapes; squares (lower line) are used for oblate particle shape. Measurements from Fig. 6 are also included (triangles, middle line). Error bars indicate the standard deviation.

for the observation data, Fig. 6) to the model calculations of Fig. 12. Results obtained with spherical rain drops (uppermost line in Fig. 13) clearly do not match the observations (middle line in Fig. 13) of -6-K PD. However, the averaged PD for oblate particles (lowest line) tends toward $-8 \mathrm{~K}$ and saturates at a brightness temperature of 180-220 K. Toward higher TB, the signal evolves back toward zero PD. The general form of the modeled PD signal is similar to the measurements but reveals a slight underestimation of PD above 150$\mathrm{K}$ brightness temperature $(-8$ instead of $-6 \mathrm{~K}$ in the observations).

This underestimation could not be confirmed as a general trend of the model results. Comparisons involving only small portions of the data were carried out. Single rain events were analyzed and were compared with a subset of the model output that matches the range of atmospheric conditions. Figure 14 illustrates an ex-

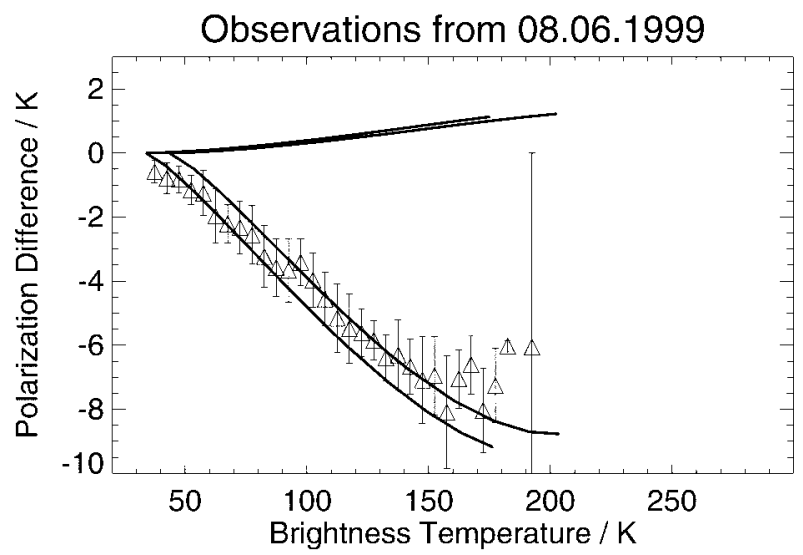

FIG. 14. Comparison of observations for 8 Jun 1999 and model results with two choices of rain-layer height $(1.5$ and $2 \mathrm{~km})$.

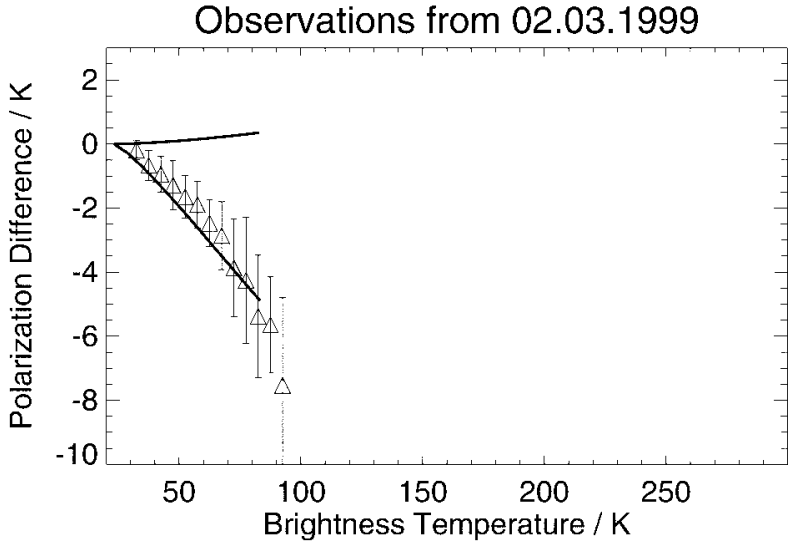

FIG. 15. Comparison of model results (1-km rain-layer height) and observations for 2 Mar 1999.

ample for observations from 8 June 1999. The recorded temperature during this rain event varies between $10.0^{\circ}$ and $13.1^{\circ} \mathrm{C}$; the relative humidity is always above $94 \%$. The model results are selected for two possible choices of the rain-layer height (1.5 and $2.0 \mathrm{~km}$, respectively). The agreement between model and measurement is fairly good except for the very last points at the high-TB end of the measurements. However, these averages are based on a very small number of radiometer observations (see Fig. 7).

Similar findings of this good agreement also can be found in winter conditions (2 March 1999; Fig. 15) as in summer conditions (24 July 1996; Fig. 16). The parameters for these days were $6.6^{\circ} \mathrm{C}$ with $1-\mathrm{km}$ rain-layer height and $16.5^{\circ}-21^{\circ} \mathrm{C}$ with 2 - and $2.5-\mathrm{km}$ rain-layer heights, respectively.

Despite these encouraging results, the conclusions drawn from such detailed comparisons are limited. The main reason for this is the fact that the most important parameter, the vertical extension of the rain column within the field of view, is not directly measured and can

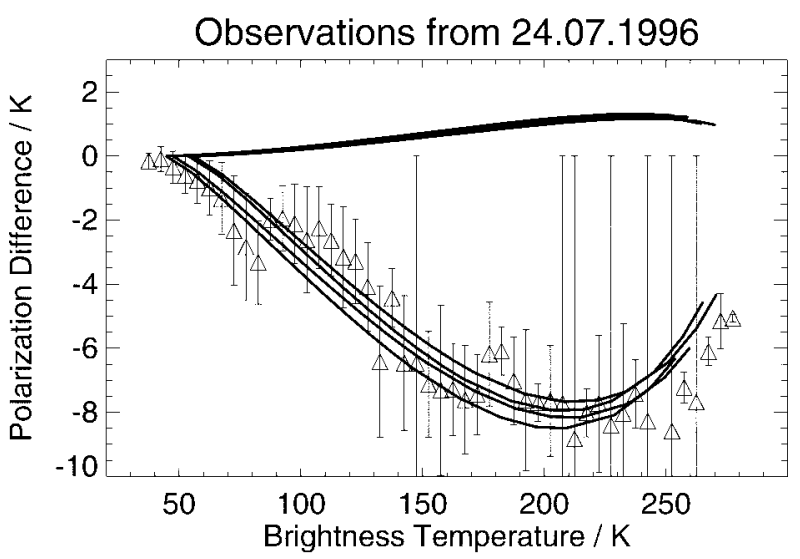

FIG. 16. Comparison of observations for 24 Jul 1996 and model results with two choices of rain-layer height $(2$ and $2.5 \mathrm{~km})$ and two atmospheric temperature profiles ( $15^{\circ}$ and $20^{\circ} \mathrm{C}$ surface temperature). 


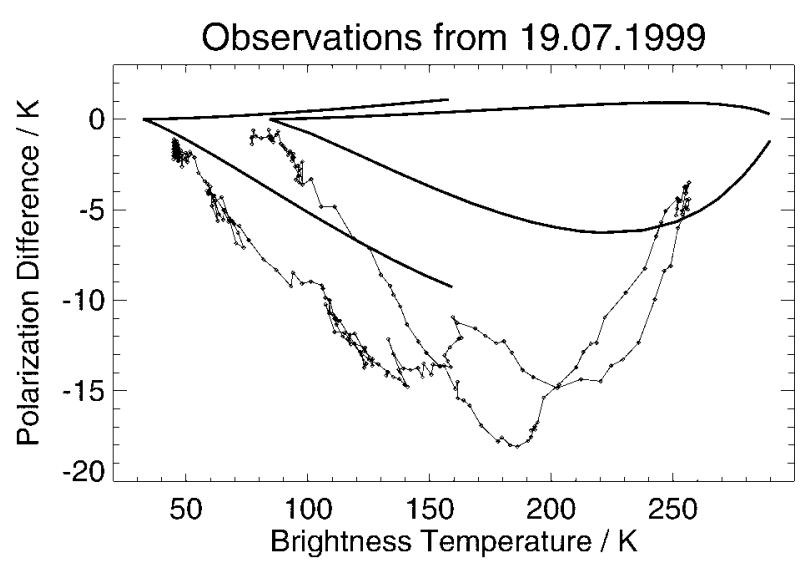

FIG. 17. Comparison of model results observations for the warm part of the rain event from 19 Jul 1999. No agreement could be reached within the given range of atmospheric parameters. This event is suspected to have a very $3 \mathrm{D}$ nature.

only be guessed. Furthermore, the precise vertical profile (of rain and cloud liquid water content) cannot be measured to such detail that a real validation could be made. These uncertainties get more important for warm surface conditions with possible rain-layer height up to the melting layer. If the freezing level, for example, is located at $3.5-\mathrm{km}$ height, then the uncertainties of the vertical profiles will be more pronounced than for winter conditions with only possible rain columns of 500-1000 m.

In addition, the limiting impact of possible three-dimensional effects (and other unidentified effects such as hail or graupel) is shown with Figs. 17 and 18. The rain event from 19 July 1999 (described earlier in Fig. 9) is broken up according to the different temperatures and humidities associated with the observations. The warmer event with temperatures ranging from $23^{\circ}$ to $29^{\circ} \mathrm{C}$ and a relative humidity between $50 \%$ and $95 \%$ shows the largest PD of all observations in this study. However, model data with this temperature and rain-layer height of $4 \mathrm{~km} \mathrm{ob-}$ viously do not fit the observations. Using the same rain rates and temperatures but a 500-m rain-layer height leads to much smaller TB and illustrates the possible range of model results for this range of ambient temperatures. Because all other choices of rain column height would lead to results in between both choices, this observation differs from the model assumptions in a very fundamental way. The observed rain rates of $36 \mathrm{~mm} \mathrm{~h}^{-1}$ suggest a convective precipitation type, which is typically of small horizontal scale and rapidly changing intensity. Such a situation generally cannot be simulated with a one-dimensional model. Geometric effects regarding the volume of rainy atmosphere intersected by the radiometer beam may be accounted for by a special setup of the vertical profile, but the multiple scattering effects of such nonisotropic conditions require a full three-dimensional treatment.

Note that the second part of this event, which occurs at $15.2^{\circ}-16.5^{\circ} \mathrm{C}$ and a rain-layer height of $2.0-2.5 \mathrm{~km}$,

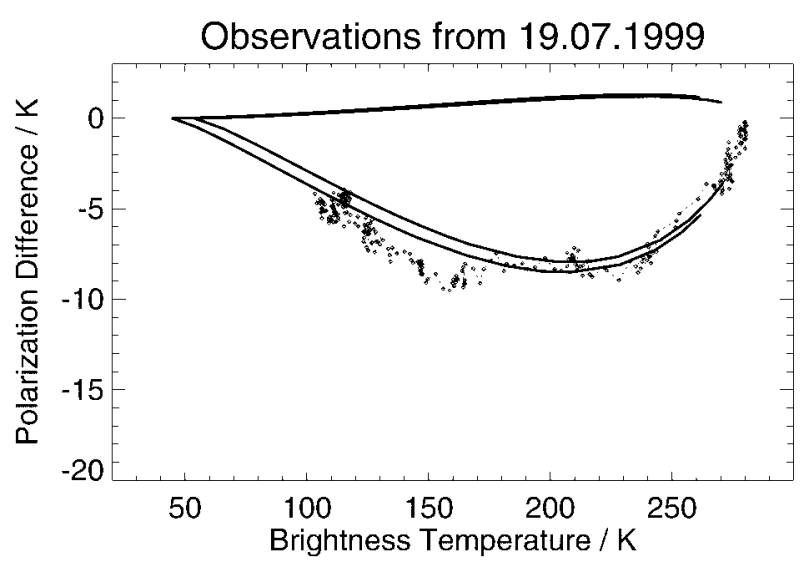

FIG. 18. Comparison of model results $\left(15^{\circ} \mathrm{C}\right.$ surface temperature; 2- and $2.5-\mathrm{km}$ rain-layer height) and observations for the cold part of the rain event from $19 \mathrm{Jul} \mathrm{1999.} \mathrm{The} \mathrm{conformance} \mathrm{for} \mathrm{this} \mathrm{more}$ stratiform precipitation is very good for the oblate drop shape.

fits very well the simulated relationship of TB and PD for such a situation (Fig. 18).

\section{Discussion}

The main purpose of this study is to answer the question of whether using the nonspherical rain drop shapes in passive microwave radiative transfer rather than using spherical particles is necessary, or if the spherical assumption is sufficiently in accordance with the measurements. When comparing the measured data (Fig. 6) with the model results after a similar data processing (Fig. 13), the agreement of experiment and model results for oblate spheroid particles is obvious. The following main features are found in observation and model data:

- decreasing PD with increasing TB,

- saturation of PD signal around 200-K brightness temperature, and

- increasing PD tending towards zero PD above $220 \mathrm{~K}$.

The general form of the PD curve as a function of TB is predicted very well by the model using nonspherical particles. Spherical particles, in contrast, produce model results for the polarization properties that are completely inconsistent with the observed data.

Nevertheless there are some differences between theoretical results for perfectly aligned spheroids and experimental data: the mean magnitude of the modeled PD signal is somewhat larger than the mean observed PD. The averaged model results produce $-8 \mathrm{~K}$ in the minimum, but the averaged observations drop only to $-6 \mathrm{~K}$. This result does not necessarily imply that the model is wrong. Although the averaged observations reveal a less pronounced PD signal than the model results, the examples from single days of observation show a very convincing conformance in signal amplitude. In rare cases, they exhibit much larger amplitudes of as much as $-18 \mathrm{~K}$. This result only proves that the 
variability of the observations is very large and that averaging over different times together with the volume integration over inhomogeneous scenes (caused by the low-elevation viewing geometry) leads to a smaller average than the average model results. The variability in the model atmospheres does not capture fully the variability of all our observations. The model uses only perfect one-dimensional rain events and thus underestimates the field-of-view averaging performed by the radiometer. From the ground-based data alone, it is practically impossible to reject all observations that might be inappropriate for this study because of their threedimensional nature.

In addition, there is, of course, still a lack of accuracy in the model used for this study. One important reason may be the idealized assumption of the perfect alignment of the hydrometeors. Natural drops will tumble around this monodisperse orientation, and the resulting distribution of orientations is expected to decrease the PD signal. Wind shear and gusts may also introduce canting angles, but it is nevertheless unphysical to assume liquid nonspherical drops to be randomly oriented: the main reasons for deformation of spherical drops to oblate drops are the vertical fall direction and gravity introducing hydrostatic pressure. Randomly oriented particles would also lead to absorption cross sections, scattering cross sections, and phase functions that are independent of incidence angle and polarization and thus would be very similar to Mie-scattering calculations for spheres. The main reason why we observe the pronounced polarization signal is that the interaction parameters differ for horizontally and vertically polarized radiation.

Further possible causes for the deviations of model results and observations are the temperature and humidity profiles. They may be inappropriate for representing the variation of the vertical profiles within all observations used for the comparison. In addition, using a vertically constant rain rate for our model calculations is a simplification of natural vertical precipitation profiles. Mixed-phase precipitation is not used in our model, but it may have an impact on the results. Because we cannot measure or derive all microphysical parameters of the vertical profile (such as temperature, humidity, liquid water content, cloud and rain particle size spectra, particle shapes, and melting-layer microphysics), it is very difficult to make a more detailed comparison of model and observations.

Another possible source of ambiguity is the choice of drop size distribution for modeling the rainfall. However, we are confident that this parameter will not totally degrade the results obtained so far, because we already have accounted partly for varying drop size distributions. The parameter of interest is the liquid water path. When producing the same LWP of a 3-km-thick rain layer with only a $1-\mathrm{km}$ rain layer, we use approximately 3 times the rain rate. Given that the slope of the Marshall-Palmer size distribution depends on the rain rate, we obtained identical LWP with different kinds of drop spectra.

An additional argument giving confidence in the comparison of model and experimental data is the fact that measurements from completely different rain events, such as winter rain, frontal rain, and convective summer rain, fit in one single plot and give a smooth curve. The short-term variability of drop size distribution and its effect on the polarization difference was investigated by Hornbostel et al. (1995, 1997). It was shown there that the drop size distribution can vary significantly for different rain events and changes even during single events. Therefore, we safely can assume a large variety of drop size distributions for all considered events. However, there may be the possibility to describe these conditions (at least for our purposes) with a simple drop size distribution, because in the long-term statistics these variations will be averaged out and will be mainly visible in the standard deviation.

For the low observation frequency of $19 \mathrm{GHz}$, the melting layer will be visible for low rain rates, especially in cold atmospheres with a low melting-layer height. We expect that the contribution to the signal from the melting layer will be substantial. Our model calculations do not explicitly account for the microphysics in the melting layer. Our expectation is that this part of the atmosphere will (under some circumstances) contain large nonspherical particles (e.g., melting snow flakes), which are likely to increase the PD signal. The missing melting layer may be a reason why the model calculations did not produce the observed PD signals below $-15 \mathrm{~K}$. On the other hand, the three-dimensional nature of a rain event may also lead to large amounts of negative PD. If a small isolated rain shaft is observed by the radiometer, then the rain-layer height is no longer of importance. Only the vertical distance, defined by the intersection of radiometer beam and the precipitation shaft, contributes to the signal. Such a situation may occur in the case of heavy convective rain. A corresponding one-dimensional model atmosphere would then be defined with a rain-layer height of only severalhundred-meters but the very large brief rain rates of a thunderstorm. This situation is likely to cause evident negative PD. However, the applicability of a one-dimensional radiative transfer model to such rain events of limited horizontal scale is at least ambiguous.

\section{Conclusions}

We compared ground-based polarized microwave observations of rainfall with simulated brightness temperature results from a one-dimensional vector radiative transfer model. The findings clearly show that using spherical particles to model microwave radiative transfer fails to explain the observations. The choice of oblate raindrop shape with size-dependent deformation and strict horizontal alignment leads to a convincing agreement for single days of observation and a small over- 
estimation of the polarization for long-term-averaged results.

We are not claiming that this description of shape and orientation (which has been exploited by radar precipitation remote sensing techniques for more than 20 years) is strictly valid and the only possible solution, but we are confident that this description is very well suited to explain the average microwave signals observed so far. The chosen representation (oblate, horizontally aligned drops) is essential for modeling the key features of the atmospheric polarization with sufficient accuracy to match the observations. Neglect of these effects by using oversimplified spherical raindrop shapes introduces significant errors into model simulations. Precise remote sensing algorithms therefore should take into account shape and alignment of rain drops.

Additional model refinements, including more detailed microphysical aspects, are expected to lead to small changes in the presented results. These effects will enhance the polarization signal (large melting particles with large nonsphericity) and diminish the polarization signal (drop oscillations, tumbling, and canting). However, the essential features of the downwelling-polarized microwave observations are well described by our radiative transfer model and have a magnitude range that cannot be neglected.

This study supports the need for remote sensing algorithms that take advantage of the information contained in the polarized scattering signal. New methods of ground-based LWP remote sensing are only one example (Czekala et al. 2001). The polarization of microwave radiation carries information about average particle size that may be used for improved rain and cloudwater retrievals with ground-based instruments.

Furthermore, the use of the more realistic hydrometeor shapes and orientation distribution functions and their full treatment in radiative transfer codes are essential even for applications that will not directly use the polarized nature of the microwave radiation. Our calculations using oblate raindrop shapes gave only small deviations from the (polarization averaged) brightness temperatures obtained with spherical particles, so the nonzero polarization difference increases the horizontally polarized intensity and decreases the vertically polarized intensity. Because most radiometers do not measure the average of two or even four components of the full Stokes vector, but rather only one polarized component, the use of such results is erroneous. They will show systematic deviations from the brightness temperatures that are connected with the total intensity of one-half the amount of observed polarization difference.

Our future research will focus on using more adequate raindrop representation by including drop oscillation and distributions of particle alignment. In addition, modeling of the mixed-phase particles in the melting layer likely is of major importance in clarifying the precise amplitudes of the polarization difference produced by raining atmospheres.

Acknowledgments. We are grateful to William B. Rossow and Michael I. Mishchenko for their support of this work. The authors acknowledge the comments of one anonymous reviewer, which helped to improve the paper. We also thank Michael I. Mishchenko for providing the T-matrix code. Part of this work is supported by the Deutsche Forschungsgemeinschaft under Grants Si-606 $1 / 1$ and Si-606 1/2.

\section{REFERENCES}

Beard, K. V., and C. Chuang, 1987: A new model for the equilibrium shape of raindrops. J. Atmos. Sci., 44, 1509-1524.

Chuang, C., and K. V. Beard, 1990: A numerical model for the equilibrium shape of electrified raindrops. J. Atmos. Sci., 47, 13741389.

Czekala, H., and C. Simmer, 1998: Microwave radiative transfer with non-spherical precipitating hydrometeors. J. Quant. Spectrosc. Radiat. Transfer, 60, 365-374.

_- S. Havemann, K. Schmidt, T. Rother, and C. Simmer, 1999: Comparison of microwave radiative transfer calculations obtained with three different approximations of hydrometeor shape. J. Quant. Spectrosc. Radiat. Transfer, 63, 545-558.

__ S. Crewell, C. Simmer, and A. Thiele, 2001: Discrimination of cloud and rain liquid water path by ground-based polarized microwave radiometry. Geophys. Res. Lett., 28, 267-270.

Evans, K. F., and G. L. Stephens, 1995: Microwave radiative transfer through clouds composed of realistically shaped ice crystals. Part I: Single scattering properties. J. Atmos. Sci., 52, 2041-2057.

—_ S. J. Walter, A. J. Heymsfield, and M. N. Deeter, 1998: Modeling of submilimeter passive remote sensing of cirrus clouds. J. Appl. Meteor., 37, 184-205.

Haferman, J. L., 1999: Microwave scattering by precipitation. Light Scattering by Nonspherical Particles: Theory, Measurements, and Applications, M. I. Mishchenko, J. W. Hovenier, and L. D. Travis, Eds., Academic Press, 481-524.

— - E. N. Anagnostou, D. Tsintikidis, W. F. Krajewski, and T. F. Smith, 1996: Physically based satellite retrieval of precipitation using a 3D passive microwave radiative transfer model. J. Atmos. Oceanic Technol., 13, 832-850.

Hansen, J. E., and L. D. Travis, 1974: Light scattering in planetary atmospheres. Space Sci. Rev., 16, 527-610.

Hornbostel, A., and A. Schroth, 1995: Propagation research on the $20 \mathrm{GHz}$ earth-Olympus satellite path. J. Electromagn. Waves Appl., 9, 439-461.

_, , and B. G. Kutuza, 1995: Polarimetric measurements and model calculations of downwelling rain brightness temperatures. Microwave Radiometry and Remote Sensing of the Environment, D. Solimini, Ed., VSP Press, 242-252.

,,$---\longrightarrow$, and A. Evtuchenko, 1997: Dual polarization and multifrequency measurements of rain rate and drop size distribution by ground-based radar and radiometers. Proc. Int. Geoscience and Remote Sensing Symp. 1997, Singapore, IEEE, $1126-1128$.

_ _ _ A. Sobachkin, B. Evtuschenko, and G. Zagorin, 1999: Passive and active Stokes vector measurements of microwave emission and scattering by precipitation. Proc. Int. Geoscience and Remote Sensing Symp. 1999, Hamburg, Germany, IEEE, 2078-2080.

Jacobson, M. D., D. C. Hoggand, and J. B. Snider, 1986: Wet reflectors in millimeter-wave radiometry-experiment and theory. IEEE Trans. Geosci. Remote Sens., 24, 784-791.

Kutuza, B., G. K. Zagorin, A. Hornbostel, and A. Schroth, 1998: Physical modeling of passive polarimetric microwave observa- 
tions of the atmosphere with respect to the third Stokes parameter. Radio Sci., 33, 677-696.

Liebe, H. J., 1985: An updated model for millimeter wave propagation in moist air. Radio Sci., 20, 1069-1089.

— , G. A. Hufford, and M. G. Cotton, 1993: Propagation modeling of moist air and suspended water/ice particles at frequencies below $1000 \mathrm{GHz}$. Preprints, 52d Specialists Meeting of the Electromagnetic Wave Propagation Panel, Palma de Mallorca, Spain, AGARD, 1-10.

Liu, Q., C. Simmer, and E. Ruprecht, 1996: Three-dimensional radiative transfer effects of clouds in the microwave spectral range. J. Geophys. Res., 101, 4289-4298.

Mie, G., 1908: Beiträge zur Optik trüber Medien, speziell kolloidaler Metallösungen (Contributions to the optics of turbid media, especially colloidal metal suspensions). Ann. Phys., 25, 377-445. [English translation 79-21946; available from National Translation Center, John Crerar Library, Chicago, IL 60616.]

Mishchenko, M. I., 2000: Calculation of the amplitude matrix for a nonspherical particle in a fixed orientation. Appl. Opt., 39, 10261031.

, L. D. Travis, and A. Macke, 1999: T-Matrix method and its applications. Light Scattering by Nonspherical Particles: Theory, Measurements, and Applications, M. I. Mishchenko, J. W. Hovenier, and L. D. Travis, Eds., Academic Press, 147-172.
Pruppacher, H. R., and K. V. Beard, 1970: A wind tunnel investigation of the internal circulation and shape of water drops falling at terminal velocity in air. Quart. J. Roy. Meteor. Soc., 96, 247256.

—_, and R. L. Pitter, 1971: A semi-empirical determination of the shape of cloud and rain drops. J. Atmos. Sci., 28, 86-94.

Rinehart, R. E., 1991: Radar for Meteorologists, Part III. 2d ed. University of North Dakota Press, $334 \mathrm{pp}$

Roberti, L., and C. Kummerow, 1999: Monte Carlo calculations of polarized microwave radiation emerging from cloud structures. J. Geophys. Res., 104, 2093-2104.

Seliga, T. A., and V. N. Bringi, 1976: Potential use of radar differential reflectivity measurements at orthogonal polarizations for measuring precipitation. J. Appl. Meteor., 15, 69-76.

Simmer, C., 1994: Satellitenfernerkundung hydrologischer Parameter der Atmosphäre mit Mikrowellen (Satellite remote sensing of hydrologic parameters of the atmosphere with microwaves). Verlag Dr. Kovac, 314 pp.

Turk, J., and J. Vivekanandan, 1995: Effects of hydrometeor shape and orientation upon passive microwave brightness temperature measurements. Microwave Radiometry and Remote Sensing of the Environment, D. Solimini, Ed., VSP Press, 187-196.

Wu, R., and J. A. Weinman, 1984: Microwave radiances from precipitating clouds containing aspherical ice, combined phase, and liquid hydrometeors. J. Geophys. Res., 89, 7170-7178. 